УДК 681.513 .5

\title{
DETERMINATION OF THE D-DOMAIN OF STABILITY OF LINEAR DYNAMICAL SYSTEMS WITH FRACTIONAL ORDER
}

\author{
O. Lobok, B. Goncharenko, N. Savitskaya \\ National University of Food Technologies \\ L. Vihrova \\ Central Ukrainian National Technical University
}

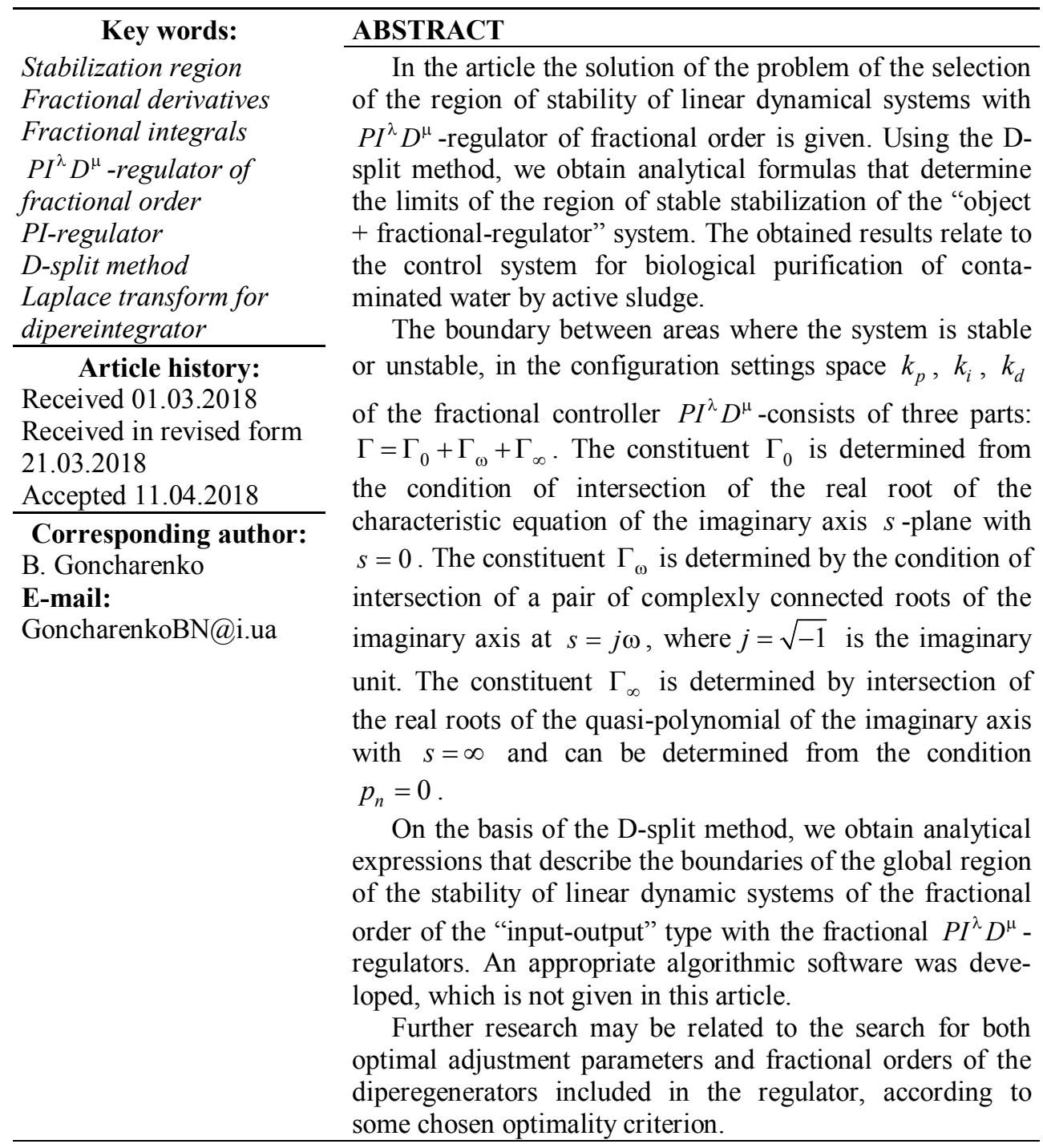

DOI: $10.24263 / 2225-2924-2018-24-2-3$ 


\title{
ВИЗНАЧЕННЯ D-ОБЛАСТІ СТІЙКОСТІ ДРОБОВИХ ЛІНІЙНИХ ДИНАМІЧНИХ СИСТЕМ
}

\author{
О.П. Лобок, Б.М. Гончаренко, Н.М. Савицька \\ Національний університет харчових технологій \\ Л.Г. Віхрова \\ Центральноукраӥнський національний технічний університет
}

У статті наведено розв'язок задачі виділення області стійкої стабілізації лінійних динамічних систем з $P I^{\lambda} D^{\mu}$-регулятором дробового порядку. Завдяки використанню методу D-розбиття отримано аналітичні формули, щуо визначають межі області стійкості системи «об'єкт» + «дробовий $P I^{\lambda} D^{\mu}$-регулятор» стосовно автоматичного керування процесом біологічного очищення забруднених вод активним мулом.

Границя між областями, де система стійка або нестійка, в просторі параметрів налаштувань $k_{p}, k_{i}, k_{d}$ дробового $P I^{\lambda} D^{\mu}$-регулятора складається з трьох частин: $\Gamma=\Gamma_{0}+\Gamma_{\omega}+\Gamma_{\infty}$. Складова $\Gamma_{0}$ визначається з умови перетину дійсним коренем характеристичного рівняння уявної осі $s$-площцни при $s=0$. Складова $\Gamma_{\omega}$ визначається з умови перетину парою комплексно сполучених коренів уявної осі при $s=j \omega$, де $j=\sqrt{-1}-$ уявна одиниця. Складова $\Gamma_{\infty}$ визначається перетином дійсними коренями квазіполінома (10) уявної осі при $s=\infty$ і може бути визначена з умови $p_{n}=0$.

На основі методу D-split отримано аналітичні вирази, щзо описують межі глобальної області стійкості лінійних динамічних систем дробового порядку mипу «вхід-вихід» з дробовими $P I^{\lambda} D^{\mu}$-регуляторами. Розроблено відповідне алгоритмічне програмне забезпечення, яке не наведено в цій статті. Оиінено ефективність результатів виділення стійкості в умовах застосування дробового $P I^{\lambda} D^{\mu}$-регулятора.

Подальші дослідження можуть бути пов'язані з пошуком як оптимальних параметрів коригування, так $і$ дробових налаштувань диферінтеграторів, включених у регулятор, відповідно до вибраного критерію оптимальності.

Ключові слова: область стабілізачиі, дробові похідні, дробові інтеграли, $P I^{\lambda} D^{\mu}$-регулятор дробового порядку, PI-регулятор, метод D-розбиття, перетворення Лапласа для диферінтегратора.

Постановка завдання. 3 початку розвитку теорії інтегро-диференціального числення дробового порядку [1] іiі перші застосування в задачах керування 3'явилися тільки близько 50 років тому [2]. Дробове числення стало ефективним інструментом для опису численних різноманітних динамічних систем. Класичні результати теорії $P I D$-регулювання поширилися на регулятори дробового порядку, які позначають як $P I^{\lambda} D^{\mu}$, де $\lambda$ i $\mu-$ 
порядки інтегрування і диференціювання сигналу похибки, які можуть мати дійсні нецілі (дробові) значення [3; 4].

Відома задача виділення глобальної області стійкості (метод $D$-розбиття) вимагала поширення на дробові динамічні системи в просторі параметрів налаштування $P I^{\lambda} D^{\mu}$-регулятора залежно від значення порядків степенів $\lambda$ i $\mu$.

Метою статті $є$ застосування методу $D$-розбиття до систем автоматичного керування технологічними процесами з дробовими регуляторами.

Викладення основних результатів дослідження. Фундаментальний опеpaтор ${ }_{a} D_{t}^{\gamma}$ часто називають диферінтегратором, який, за визначенням Грюнвальда-Летникова, для порядку $\gamma$ має вигляд:

$$
{ }_{a} D_{t}^{\gamma} f(t)=\lim _{h \rightarrow 0} \frac{1}{h^{\gamma}} \sum_{j=0}^{[(t-a) / h]}(-1)^{j}\left(\begin{array}{l}
\gamma \\
j
\end{array}\right) f(t-j h),
$$

де $\left(\begin{array}{l}\gamma \\ j\end{array}\right)=\frac{\Gamma(\gamma+1)}{\Gamma(j+1) \Gamma(\gamma-j+1)} ; \Gamma(x)$ - гама функція Ейлера; $h>0$ - приріст часової координати; $f(x)$ - функція, до якої застосовується оператор диферінтегрування; [·] — означає цілу частину числа. Це визначення показує, що цілочисельні похідні вимагають використання кінцевих рядів, а дробові нескінченного числа членів ряду.

Доведено [5], що перетворення Лапласа, яке $є$ основою визначення поняття передавальної функції, для диферінтегратора має вигляд:

$$
L\left\{{ }_{0} D_{t}^{\gamma} f(t)\right\}=\int_{0}^{\infty} e_{0}^{-s t} D_{t}^{\gamma} f(t) d t=s^{\gamma} F(s)-\left.\sum_{j=0}^{n-1} s^{j}(-1)_{0}^{j} D_{t}^{\gamma-j-1} f(t)\right|_{t=0},
$$

де $F(s)=L\{f(t)\}$ — звичайне перетворення Лапласа функції $f(x) ; n$ - ціле число, яке задовольняє умову $n-1<\gamma \leq n$.

Відзначимо, що якщо $\left.{ }_{0} D_{t}^{\gamma-j-1} f(t)\right|_{t=0}=0, j=0,1,2, \ldots, n-1$, то 3 (2) випливає, що $L\left\{{ }_{0} D_{t}^{\gamma} f(t)\right\}=s^{\gamma} F(s)$.

Передавальна функція дробового порядку задається таким виразом:

$$
G(s)=\frac{N(s)}{D(s)}=\frac{b_{n} s^{\beta_{n}}+b_{n-1} s^{\beta_{n-1}}+\ldots+b_{1} s^{\beta_{1}}+b_{0} s^{\beta_{0}}}{a_{n} s^{\alpha_{n}}+a_{n-1} s^{\alpha_{n-1}}+\ldots+a_{1} s^{\alpha_{1}}+a_{0} s^{\alpha_{0}}}=\frac{\sum_{i=0}^{n} b_{i} s^{\beta_{i}}}{\sum_{i=0}^{n} a_{i} s^{\alpha_{i}}}
$$

де $a_{i}, b_{i}, \beta_{n}>\beta_{n-1}>\ldots>\beta_{1}>\beta_{0} \geq 0, \alpha_{n}>\alpha_{n-1}>\ldots>\alpha_{1}>\alpha_{0} \geq 0$ - довільні дійсні числа.

У часовій області передавальній функції (3) відповідає неоднорідне диференціальне рівняння дробового порядку виду: 


$$
\sum_{i=0}^{n} a_{i} D^{\alpha_{i}} y(t)=\sum_{i=0}^{n} b_{i} D^{\beta_{i}} u(t),
$$

де $y(t)$ - вихід, а $u(t)$ - вхід об'єкта керування; ${ }_{a} D_{t}^{\gamma}-$ диферінтегратор.

Передавальна функція дробового $P I^{\lambda} D^{\mu}$-регулятора має вигляд:

$$
C(s)=k_{p}+k_{i} s^{-\lambda}+k_{d} s^{\mu},
$$

де $\lambda$ и $\mu-$ дробові порядки, значення яких належить до області $(0,2) ; k_{p}$, $k_{i}, k_{d}$ - налаштовувальні параметри регулятора.

У часовій області передавальній функції (5) відповідає керування виду

$$
u(t)=k_{p} \cdot e(t)+k_{i} \cdot\left({ }_{0} D_{t}^{-\lambda} e(t)\right)+k_{d} \cdot\left({ }_{0} D_{t}^{\mu} e(t)\right),
$$

де - ${ }_{0} D_{t}^{\gamma}$, як зазначалося вище, диферінтегратор.

Відповідно до означеної мети статті підмета дослідження полягає в тому, щоб віднайти методом $D$-розбиття область стійкості динамічної системи «об'єкт + регулятор» при допустимих значеннях параметрів налаштувань $k_{p}, k_{i}, k_{d}$ дробового $P I^{\lambda} D^{\mu}$-регулятора, які стабілізують об'єкт керування.

Це важливо і при конструюванні $P I^{\lambda} D^{\mu}$-регуляторів, і для пошуку оптимальних налаштувань регуляторів на знайденій параметричній області стабілізації за обраним критерієм.

Передавальна функція замкненої системи «об’єкт + регулятор» має загальний вигляд:

$$
W(s)=\frac{C(s) G(s)}{1+C(s) G(s)}=\frac{Q(s)}{P(s)},
$$

де

$$
\begin{gathered}
Q(s)=\sum_{j=0}^{n}\left[k_{p} b_{j} s^{\lambda+\beta_{j}}+k_{i} b_{j} s^{\beta_{j}}+k_{d} b_{j} s^{\lambda+\mu+\beta_{j}}\right] ; \\
P(s)=\sum_{j=0}^{n}\left[a_{j} s^{\lambda+\alpha_{j}}+k_{p} b_{j} s^{\lambda+\beta_{j}}+k_{i} b_{j} s^{\beta_{j}}+k_{d} b_{j} s^{\lambda+\mu+\beta_{j}}\right] .
\end{gathered}
$$

Область стійкості, яку позначимо через $S$, в просторі параметрів знаходиться за умови приналежності до лівої напівплощини комплексної $s$-площини всіх дійсних частин коренів характеристичного квазіполінома $P(s)$, який для зручності подамо у вигляді:

$$
P(s)=\sum_{j=0}^{n} p_{j} s^{q_{j}}=p_{n} s^{q_{n}}+p_{n-1} s^{q_{n-1}}+\ldots+p_{1} s^{q_{1}}+p_{0} s^{q_{0}},
$$

де $q_{j}$ - впорядковані дробові порядки степенів, причому $q_{n}>q_{n-1}>\ldots>q_{0}$; $p_{j}$ - коефіцієнти, які визначаються коефіцієнтами передавальної функції 
об’єкта керування і параметрами налаштувань $k_{p}, k_{i}, k_{d}$ дробового $P I^{\lambda} D^{\mu}$ регулятора.

Для виділення області стійкості системи (об'єкта керування з регулятором) використовуємо метод $D$-розбиття простору параметрів [6]. Нагадаємо, що згідно з цим методом границя між областями, де система (стійка або нестійка), в просторі параметрів налаштувань утворюється трьома частинами: $\Gamma=\Gamma_{0}+\Gamma_{\omega}+\Gamma_{\infty}$.

Складова $\Gamma_{0}$ визначається з умови перетину дійсним коренем характеристичного рівняння уявної осі $s$-площини при $s=0$. Тобто складову $\Gamma_{0}$ знаходять шляхом підстановки $s=0$ в рівняння $P(s)=0$, де $P(s)$ визначається рівнянням (10). Звідси випливає, що $\Gamma_{0}$ може бути визначена з умови $p_{0}=0$, якщо значення найменшого порядку $q_{0}$ дорівнює 0 , тобто при $s^{q_{0}}=1$. Якщо $q_{0} \neq 0$, тобто $s^{q_{0}} \neq 1$, то границі $\Gamma_{0}$ не існує. Складова $\Gamma_{\omega}$ визначається 3 умови перетину парою комплексно сполучених коренів уявної осі при $s=j \omega$, де $j=\sqrt{-1}-$ уявна одиниця. В цьому випадку квазіполіном (10) стає нестійким і дійсна й уявна частини рівняння $P(j \omega)=0$ починають дорівнювати нулю одночасно.

Складова $\Gamma_{\infty}$ визначається перетином дійсними коренями квазіполінома (10) уявної осі при $s=\infty$ і може бути визначена з умови $p_{n}=0$.

Застосовуючи ці передумови до досліджуваної системи «об'єкт + регулятор» і аналізуючи характеристичний квазіполіном (9), приходимо до висновку, що складові $\Gamma_{0}$ та $\Gamma_{\infty}$ границі області стійкості являють собою прямі лінії:

$$
\begin{gathered}
\Gamma_{0} \text {-лінія: }\left\{\begin{array}{l}
k_{i}=0, \quad \text { при } s^{\beta_{0}}=1, \\
\text { не існує, при } s^{\beta_{0}} \neq 1,
\end{array}\right. \\
\Gamma_{\infty} \text { - лінія: } \begin{cases}k_{d}=0, & \text { при }\left(\alpha_{n}=\beta_{n}\right) \text { або }\left(\alpha_{n}>\beta_{n} \text { i } \mu>\alpha_{n}-\beta_{n}\right), \\
k_{d}=-a_{n} / b_{n}, & \text { при }\left(\alpha_{n}>\beta_{n} \text { i } \mu=\alpha_{n}-\beta_{n}\right), \\
\text { не існує, } & \text { при }\left(\alpha_{n}>\beta_{n} \text { i } \mu<\alpha_{n}-\beta_{n}\right) .\end{cases}
\end{gathered}
$$

Для побудови складової $\Gamma_{\omega}$ підставим $s=j \omega$ в рівняння $P(s)=0$, де $P(s)-$ квазіполіном (9). Тоді отримаємо:

$$
\begin{gathered}
P(j \omega)=\sum_{j=0}^{n}\left[a_{j}(j \omega)^{\lambda+\alpha_{j}}+k_{p} b_{j}(j \omega)^{\lambda+\beta_{j}}+k_{i} b_{j}(j \omega)^{\beta_{j}}+k_{d} b_{j}(j \omega)^{\lambda+\mu+\beta_{j}}\right]= \\
=\operatorname{Re}\{P(j \omega)\}+j \cdot \operatorname{Im}\{P(j \omega)\}=0,
\end{gathered}
$$

де $\operatorname{Re}\{P(j \omega)\}$ та $\operatorname{Im}\{P(j \omega)\}$ означають, відповідно, дійсну та уявну частини квазіполінома $P(j \omega)$. 
Для подальшого перетворення виразу (11) пригадаємо, що нецілий степінь комплексного числа $(\sigma+j \omega)^{\gamma}$ може бути вирахований за формулою МуавраЛапласа:

$$
(\sigma+j \omega)^{\gamma}=\left(\sigma^{2}+\omega^{2}\right)^{\gamma / 2}[\cos (\gamma \varphi)+j \sin (\gamma \varphi)],
$$

де $\varphi=\arctan (\omega / \sigma) ; \sigma-$ дійсна частина, $\omega-$ уявна частина; $\gamma$ дробовий порядок комплексного числа.

Вираз $j^{\gamma}$ у рівнянні (11) може бути представлений згідно з формулою (12) так:

$$
j^{\gamma}=\cos \left(\frac{\pi}{2} \gamma\right)+j \sin \left(\frac{\pi}{2} \gamma\right)
$$

Далі, прирівнявши до нуля дійсну і уявну частину рівняння (11), з урахуванням формули (13) отримаємо:

$$
\left\{\begin{array}{l}
\operatorname{Re}\{P(j \omega)\}=k_{p} R_{1 p}(\omega)+k_{i} R_{1 i}(\omega)+k_{d} R_{1 d}(\omega)+H_{1}(\omega)=0, \\
\operatorname{Im}\{P(j \omega)\}=k_{p} R_{2 p}(\omega)+k_{i} R_{2 i}(\omega)+k_{d} R_{2 d}(\omega)+H_{2}(\omega)=0
\end{array}\right.
$$

де

$$
\begin{gathered}
R_{1 p}(\omega)=\sum_{j=0}^{n} b_{j} \omega^{\lambda+\beta_{j}} \cos \left(\frac{\pi}{2}\left(\lambda+\beta_{j}\right)\right), R_{1 i}(\omega)=\sum_{j=0}^{n} b_{j} \omega^{\beta_{j}} \cos \left(\frac{\pi}{2} \beta_{j}\right), \\
R_{1 d}(\omega)=\sum_{j=0}^{n} b_{j} \omega^{\lambda+\mu+\beta_{j}} \cos \left(\frac{\pi}{2}\left(\lambda+\mu+\beta_{j}\right)\right), H_{1}(\omega)=\sum_{j=0}^{n} a_{j} \omega^{\lambda+\alpha_{j}} \cos \left(\frac{\pi}{2}\left(\lambda+\alpha_{j}\right)\right), \\
R_{2 p}(\omega)=\sum_{j=0}^{n} b_{j} \omega^{\lambda+\beta_{j}} \sin \left(\frac{\pi}{2}\left(\lambda+\beta_{j}\right)\right), R_{2 i}(\omega)=\sum_{j=0}^{n} b_{j} \omega^{\beta_{j}} \sin \left(\frac{\pi}{2} \beta_{j}\right), \\
R_{2 d}(\omega)=\sum_{j=0}^{n} b_{j} \omega^{\lambda+\mu+\beta_{j}} \sin \left(\frac{\pi}{2}\left(\lambda+\mu+\beta_{j}\right)\right), H_{2}(\omega)=\sum_{j=0}^{n} a_{j} \omega^{\lambda+\alpha_{j}} \sin \left(\frac{\pi}{2}\left(\lambda+\alpha_{j}\right)\right) .
\end{gathered}
$$

Система лінійних рівнянь (14) містить більше невідомих $\left(k_{p}, k_{i}, k_{d}\right)$, ніж число рівнянь, тому для однозначного іiї розв'язання один із параметрів системи мусить бути обраний довільно. Якщо за такий параметр обрати коефіцієнт $k_{p}$, то система (14) стає системою лінійних алгебраїчних рівнянь другого порядку щодо невідомих $k_{i}$ та $k_{d}$, розв'язок якої має вигляд:

$$
\begin{gathered}
k_{i}=\frac{\Delta_{i}(\omega)}{\Delta(\omega)}, k_{d}=\frac{\Delta_{d}(\omega)}{\Delta(\omega)} \\
\Delta_{i}(\omega)=R_{1 d}(\omega) H_{2}(\omega)-R_{2 d}(\omega) H_{1}(\omega)+k_{p}\left(R_{1 d}(\omega) R_{2 p}(\omega)-R_{1 p}(\omega) R_{2 d}(\omega)\right), \\
\Delta_{d}(\omega)=R_{2 i}(\omega) H_{1}(\omega)-R_{1 i}(\omega) H_{2}(\omega)+k_{p}\left(R_{1 p}(\omega) R_{2 i}(\omega)-R_{1 i}(\omega) R_{2 p}(\omega)\right),
\end{gathered}
$$




$$
\Delta(\omega)=R_{1 i}(\omega) R_{2 d}(\omega)-R_{1 d}(\omega) R_{2 i}(\omega)=\omega^{\lambda+\mu} \sin \left(\frac{\pi}{2}(\lambda+\mu)\right)\left(R_{1 i}^{2}(\omega)+R_{2 i}^{2}(\omega)\right) .
$$

Застосуємо тепер ці результати для виділення області стійкості системи керування біологічним очищенням забруднених вод активним мулом 3 дробовим $P I^{\lambda} D^{\mu}$-регулятором. За припущення, що кінетика процесу зростання біомаси описується рівнянням Моно [7], у [8] була отримана лінеаризована модель біоочисної системи «аеротенк + відстійник» у вигляді математичної моделі з одним входом і одним виходом:

$$
\frac{d \boldsymbol{x}(t)}{d t}=A x(t)+b u(t), y(t)=x_{2}(t)=c^{T} \boldsymbol{x}(t),
$$

де $\boldsymbol{x}(t)=\left(x_{1}(t), x_{2}(t), x_{3}(t)\right)^{T}$ - вектор стану, в якому $x_{1}(t), x_{2}(t)-$ відповідно, концентрація біомаси і субстрату в аеротенку; $x_{3}(t)$ - концентрація рециркулюючої біомаси з відстійника в біореактор-аеротенк; $u(t)-$ одномірна функція керування - швидкість розведення (аналог об'ємної швидкості потоку); $y(t)$ - спостережуваний вихід системи - концентрація субстрату.

Системна матриця $A$ і вектори $b$ и $c$ в моделі (17) визначаються таким чином:

$$
A=\left(\begin{array}{lll}
a_{11} & a_{12} & a_{13} \\
a_{21} & a_{22} & a_{23} \\
a_{31} & a_{32} & a_{33}
\end{array}\right), b=\left(\begin{array}{l}
b_{1} \\
b_{2} \\
b_{3}
\end{array}\right), c=\left(\begin{array}{l}
0 \\
1 \\
0
\end{array}\right),
$$

де

$$
\begin{gathered}
a_{1,1}=\mu_{\max } \frac{x_{2}^{*}}{k_{s}+x_{2}^{*}}-(1+r) u^{*}, a_{1,2}=\mu_{\max } k_{s} \frac{x_{1}^{*}}{\left(k_{s}+x_{2}^{*}\right)^{2}}, a_{1,3}=r u^{*}, \\
a_{2,1}=-\frac{\mu_{\max }}{Y} \frac{x_{2}^{*}}{k_{s}+x_{2}^{*}}, a_{2,2}=-\frac{\mu_{\max } k_{s}}{Y} \frac{x_{1}^{*}}{\left(k_{s}+x_{2}^{*}\right)^{2}}-(1+r) u^{*}, a_{2,3}=0, \\
a_{3,1}=(1+r) u^{*}, a_{3,2}=0, a_{3,3}=-(\beta+r) u^{*}, \\
b_{1}=-(1+r) x_{1}^{*}+r x_{3}^{*}, b_{2}=-(1+r) x_{2}^{*}+s_{i n}, b_{3}=-(\beta+r) x_{3}^{*}+(1+r) x_{1}^{*} .
\end{gathered}
$$

Тут позначено: $u^{*}$ - задане номінальне керування, $x^{*}=\left(x_{1}^{*}, x_{2}^{*}, x_{3}^{*}\right)^{T}-$ відповідний йому обчислений вектор рівноважного стану; $\mu_{\max }-$ максимальна питома швидкість росту біомаси; $k_{s}$ - константа насичення, що визначається експериментальним шляхом; $s_{i n}$ - концентрація субстрату у вхідному потоці; $Y$ - чинник виходу (прибутковості) біомаси; $r, \beta-$ коефіцієнти, що визначають, відповідно, відношення рециркуляційного потоку і потоку відходів біомаси до вхідного потоку.

У частотній області модель (17) може бути представлена у вигляді: 


$$
Y(s)=G(s) U(s),
$$

де $U(s), Y(s)$ - перетворення Лапласа, відповідно, входу і виходу; $G(s)$ передавальна функція об'єкта керування.

$$
G(s)=c^{T}(s E-A)^{-1} b=\frac{c^{T} a d j(s E-A) b}{\operatorname{det}(s E-A)}=\frac{p_{2} s^{2}+p_{1} s+p_{0}}{s^{3}+q_{2} s^{2}+q_{1} s+q_{0}} .
$$

Тут через $\operatorname{adj}(s E-A)$ позначена приєднана матриця матриці $s E-A$, а коефіцієнти поліномів чисельника і знаменника $p_{i}, q_{i}$ обчислюються за формулами:

$$
\begin{gathered}
p_{0}=b_{2} a_{11} a_{33}-b_{1} a_{21} a_{33}-b_{2} a_{13} a_{31}, q_{0}=a_{12} a_{21} a_{33}+a_{13} a_{31} a_{22}-a_{11} a_{22} a_{33}, \\
p_{1}=b_{1} a_{21}-b_{2} a_{11}-b_{2} a_{33}, q_{1}=a_{11} a_{22}+a_{11} a_{33}+a_{22} a_{33}-a_{13} a_{31}-a_{12} a_{21}, \\
p_{2}=b_{2}, q_{2}=-a_{11}-a_{22}-a_{33} .
\end{gathered}
$$

Якщо керування $u(t)$ в часовій області конструювати в класі дробових $P I^{\lambda} D^{\mu}$-регуляторів виду (5)

$$
u(t)=-\left(k_{p} \cdot y(t)+k_{i} \cdot\left({ }_{0} D_{t}^{-\lambda} y(t)\right)+k_{d} \cdot\left({ }_{0} D_{t}^{\mu} y(t)\right)\right),
$$

то передавальна функція системи «процес біоочищення + регулятор» буде визначатися виразом $W(s)=Q(s) / P(s)$, де $Q(s)=C(s) G(s), \quad P(s)=1+C(s) G(s)$, $C(s)$ - передавальна функція дробового регулятора, що визначається за формулою (5); $G(s)$ - передавальна функція об'єкта керування, що обчислюється за формулами (18), (19).

Для визначення області допустимих значень параметрів налаштування $k_{p}, k_{i}, k_{d}$ дробового $P I^{\lambda} D^{\mu}$-регулятора, який стабілізує роботу біоочисної системи, використовувалися розрахункові формули (15), (16), що описують границі областей стійкості системи 3 дробовим регулятором. Обчислювальні експерименти для виявлення графічної залежності області стійкості від параметрів налаштування дробового регулятора проводилися в середовищі математичної системи MATLAB і будуть розглянуті в окремій статті.

\section{Висновки}

На основі методу $D$-розбиття отримані аналітичні вирази, які описують границі глобальної області стійкості лінійних динамічних систем дробового порядку типу «вхід-вихід» 3 дробовими $P I^{\lambda} D^{\mu}$-регуляторами. Області стійкості побудовані на основі обчислювальних експериментів у просторі параметрів налаштування дробових $P I^{\lambda} D^{\mu}$-регуляторів при фіксованих порядках диферінтеграторів у складі регулятора. Розроблене відповідне алгоритмічнопрограмне забезпечення, яке в цій статті не приведене. Очевидна можливість застосування методу до будь-яких лінійних об'єктів, динаміка яких описується рівнянням структури (17), а обмеження методу може бути пов'язане 3 
нелінійністю або 3 невизначеністю. Подальші дослідження можуть бути пов'язані з пошуком як оптимальних параметрів налаштування, так і дробових порядків диферінтеграторів, що входять у регулятор, згідно 3 деяким обраним критерієм оптимальності, а також і з виявленням графічної залежності $D$-області стійкості від параметрів $k_{p}, k_{i}, k_{d}$ налаштування дробового регулятора.

\section{Література}

1. Podlubny I. Fractional Differential Equations / Mathematics in Sciences and Engineering, Vol. 198. — Academic Press, 1999. - 340 p.

2. Tustin A., Allason J.T., Layton J.M., Jakeways R.J.: The design of systems for automatic control of the position of massive object. / Proc. Inst. Electr. Eng. 105 (C-1), 1958, pp. 1-57.

3. Podlubny I. Fractional-order systems and PID controllers. IEEE Transactions on Automatic Control, 1999, vol. 44, pp. 208-214.

4. Бутковский А.Г. Дробное интегро-дифференциальное исчисление и его приложения в теории управления. II. Дробные динамические системы: моделирование и аппаратная реализация / А.Г. Бугковский, С.С. Постнов, Е.А. Постнова // Автоматика и телемеханика.2013. - № 5. - С. 3-34.

5. Учайкин В.В. Метод дробных производных. - Ульяновск, Артишок, 2008. - 512 с.

6. Hamamci S.E., Tan N. Design of PI controllers for achieving time and frequency domain specifications simultaneously / ISA Trans. Vol. 45 (4), 2006, pp. 529-543.

7. Nejjari F., Roux G, Dahhou B, Benhammou A. Estimation and optimal control design of a biological wastewater treatment process / Mathematics and computers in simulation, vol. 48, 1999, pp. 269-280.

8. Лобок О.П. Моделювання оптимального автоматичного керування процесом біологічної очистки забруднених вод регуляторами дробового порядку / О.П. Лобок, Б.М. Гончаренко, М.А. Сич, Л.Г. Віхрова // Збірник наук. праць Кіровоградського національного технічного університету. Техніка в сільськогосподарському виробництві, галузеве машинобудування, автоматизація. — Кропивницький : КНТУ. — 2017. — № 30. — С. 152-160. 